\title{
MULTI-DOMAIN SPECTRAL APPROACH WITH SOMMERFELD CONDITION FOR THE MAXWELL EQUATIONS
}

\author{
CHRISTIAN KLEIN AND NIKOLA STOILOV
}

\begin{abstract}
We present a multi-domain spectral approach with an exterior compactified domain for the Maxwell equations for monochromatic fields. The Sommerfeld radiation condition is imposed exactly at infinity being a finite point on the numerical grid. As an example, axisymmetric situations in spherical and prolate spheroidal coordinates are discussed, as well as the interaction of a radiating dipole with a nano-particle.
\end{abstract}

\section{Introduction}

The interaction between electromagnetic radiation and matter is arguably one of the most important problems of physics, and one of great practical importance. The governing equations for this are the Maxwell equations, and their efficient numerical solution in situations appearing in applications is thus crucial. Interestingly the classical Maxwell equations are also relevant in the context of quantum emitters since the latter can be treated as a quantum system interacting with a classical field, see for instance [11, 28] and references therein. In general one is not interested in the solution of a particular initial value problem in this context, but a discussion in the frequency domain. In this case, the Maxwell equations in non-magnetizable matter can be cast into the form (see section 2 for a short derivation and for references) of a vector Helmholtz equation,

$$
\nabla \times \nabla \times \mathbf{E}(\mathbf{x}, \omega)-\omega^{2} \epsilon(\mathbf{r}, \omega) \mathbf{E}(\mathbf{x}, \omega)=\mathbf{f} .
$$

Here $\mathbf{x} \in \mathbb{R}^{3}$ with components $x_{i}, i=1,2,3, \mathbf{E} \in \mathbb{C}^{3}$ is the electric field, $\omega \in \mathbb{R}$ is the frequency, $\epsilon(\mathbf{r}, \omega)$ is the permittivity in the Maxwell equations with matter, see (4) and (5), $\mathrm{f}$ is an inhomogenity due to free currents, and $\nabla$ is the vector operator with components $\nabla_{i}=\frac{\partial}{\partial x_{i}}$,

Date: January 20, 2021.

We thank the anonymous referees for helpful suggestions and remarks. This work is partially supported by the ANR-FWF project ANuI - ANR-17-CE40-0035, the isite BFC project NAANoD, the EIPHI Graduate School (contract ANR-17-EURE0002), by the European Union Horizon 2020 research and innovation program under the Marie Sklodowska-Curie RISE 2017 grant agreement no. 778010 IPaDEGAN and the EITAG project funded by the FEDER de Bourgogne, the region BourgogneFranche-Comté and the EUR EIPHI.. 
$i=1,2,3$. It is the goal of this paper to provide a multi-domain spectral method for the solution of this equation for systems corresponding to a localized isolated matter configuration thus extending the method by Gharti et al [15]- [19].

In order to get a unique solution to the Helmholtz equation (1), a condition needs to be imposed at infinity. Sommerfeld suggested that there should be no incoming radiation at infinity, i.e., the only source of radiation should be the matter distribution. The Sommerfeld radiation condition [29] reads

$$
\lim _{\|\mathbf{x}\| \rightarrow \infty}\|\mathbf{x}\|\left(e^{i \omega\|\mathbf{x}\|} \mathbf{E}(\mathbf{x}, \omega)-1\right)=0,
$$

where $\|\cdot\|$ is the euclidean norm (in vacuum $\epsilon(\mathbf{x}, \omega)=1$ ). This implies, however, that the solution has an oscillatory singularity (proportional to $e^{i \omega|| \mathbf{x} \|}$ ) for large $\|\mathbf{x}\|$ which is numerically challenging. What makes things worse is that it is known, see [2, 34], that the solution of the scalar Helmholtz equation

$$
\Delta E+\omega^{2} E=0,
$$

where $\Delta$ is the three-dimensional Laplace operator, with a Sommerfeld condition has in spherical coordinates (see section 2.1) for large $r$ the form

$$
E=\frac{e^{-i \omega r}}{\omega r} \sum_{n=0}^{\infty} \frac{a_{n}(\theta, \phi)}{(\omega r)^{n}},
$$

where the $a_{n}, n \in \mathbb{R}$ depend only on $\theta, \phi$. This means that the solution is not only oscillatory near infinity, but also slowly decaying in $1 / r$.

Imposing boundary conditions at infinity has been discussed in many works and in various contexts. The most popular approach is to truncate the problem and solve it on a finite domain by imposing artificial boundaries at a finite distance from the physical system (for a general review see e.g. [20, 32] and references therein). One option in this case is to consider non-reflecting boundaries (NRBs), that is, boundaries that allow the waves to leave the truncated domain without spurious reflections that may pollute the solution in the computational domain of interest. There are two main groups of NRBs, namely, Non-Reflecting Boundary Conditions (NRBCs) and Non-Reflecting Boundary Layers (NRBLs). NRBCs are boundary conditions on the artificial boundary that absorb incident waves, see for example [3] [13]. On the other hand, NRBLs are additional computational domains which absorb waves that are traveling inside the layer, effectuating trivial boundary condition at the end of the domain towards infinity. The most prominent among the NRBL techniques is the Perfectly Matched Layer (PML) initially developed by Bérenger in 1994 for electromagnetic scattering [6]. The idea is to add an absorbing layer so that plane waves produce no reflection and that inside the layer the solution decays exponentially [21]. 
Such approaches work very well for linear problems as studied here, but need in general an optimization of the parameters of the absorbing layer, see for instance the discussion in [4]. On the other hand in the case of slowly decaying potentials as in electromagnetism and relativity approximate implementation of non-reflecting boundary conditions can lead to non-negligible errors, see for instance the comparison of compactification (as in the present paper) and truncation schemes in a relativistic context in [12]. In a time dependent context, see [27] for an implementation of the Sommerfeld condition in this context, for compactification approaches based on the conformal invariance the review [14]. Note that the techniques explained in this paper can be directly applied to similar problems in linearized gravity.

Another approach, which is closely connected to the one taken in the present work, is that of mapped infinite elements Its origin can be traced back to the works of Zienkevich and Bettess [37], for a comprehensive review see [8]. The basic idea in one dimension, $x$, is to add an element extending to infinity, where we map to a new coordinate so that $x=2 x_{0} /(1-\xi)$. The infinite element is thus mapped onto $[-1,1]$ and infinity becomes the regular grid point $\xi=1$. In a general setting, shape functions $M_{i}$ in the infinite element are polynomials in $(\xi)$, which translates to polynomials in $1 / x$. The Sommerfeld condition can be directly implemented on the shape functions and take them in the form $M_{i}=e_{1}^{-i \omega x} P_{i}(\xi)$. The idea was further developed by Beer and Meeks in [5]. It was applied under the name "infinite boundary element" for electromagnetic and other problems by Kagawa et al [22]. Recently this was developed into 'spectral infinite element' methods by Gharti et al. [15, 16] when dealing with geophysical problems.

In this paper we $_{2}$ use an approach similar to the mapped infinite elements [37, 8] for of a multi-domain spectral approach. Note that spectral methods are distinguished by their excellent approximation properties for analytical functions since the numerical error in such a case decreases exponentially with the numerical resolution. They are thus especially effective if the function $\epsilon(\mathbf{x}, \omega)$ in (11) is analytical in the considered domains. We concentrate here on the case where this is true on concentric spheres or spheroids, for instance a spherical or spheroidal conductor in vacuum, possibly with multiple layers. More precisely, we consider a number $N_{d}+1, N_{d} \in \mathbb{N}$, of domains where $\epsilon$ is smooth in each of them, but in concrete examples we discuss the case of three domains, an interior domain such that the boundary of the matter is a domain boundary, a second domain in vacuum 1 , and a third domain with the local parameter $1 /\|\mathbf{x}\|$ around infinity. As in

\footnotetext{
${ }^{1}$ Experience in an astrophysical context in [35] shows that it is numerically recommended not to match the infinite domain directly to the matter configuration, but to apply an intermediate domain.
} 
[37, 8] we split off the oscillatory term in (3) by writing

$$
E=e^{-i \omega r} \tilde{E}
$$

and solving the equations in the compactified domain for $\tilde{E}$ which is non-oscillatory and analytical in $1 /\|\mathbf{x}\|$ and thus ideally suited for a spectral method.

The paper is organized as follows: in section 2 , we review the Maxwell equations in spherical and prolate spheroidal coordinates and introduce the twist potential in the axisymmetric case. In section 3 we discuss the matching and the Sommerfeld radiation condition. In section 4 we present our numerical approach. Some examples are discussed in section 5. We add some concluding remarks in section 6 .

Notation: Partial derivatives of a function $u$ with respect to $x$ are denoted by $\partial_{x} u$ or $u_{x}$, vector indices are superscripts.

\section{The Maxwell equations in spherical and prolate SPHEROIDAL COORDINATES}

In this section we give a brief summary of the Maxwell equations in matter. A convenient form for a numerical solution is presented in the axisymmetric case in spherical and prolate spheroidal coordinates.

Throughout this paper we assume that the studied problems allow a Fourier transform in $t$ with $\omega$ being the dual Fourier variable to $t$. This means we are interested in the long time interaction between electromagnetic radiation rather than in specific initial value problems. The Maxwell equations in this case read

$$
\nabla \cdot \mathbf{D}(\mathbf{x}, \omega)=\sigma, \quad \nabla \cdot \mathbf{H}(\mathbf{x}, \omega)=0,
$$

$$
\text { (4) } \nabla \times \mathbf{E}(\mathbf{x}, \omega)=-i \omega \mathbf{B}(\mathbf{x}, \omega), \quad \nabla \times \mathbf{H}(\mathbf{x}, \omega)=\mathbf{J}+i \omega \mathbf{D}(\mathbf{x}, \omega) \text {, }
$$

where $\sigma$ is the density of the free charges, and $\mathbf{J}$ is the density of the free currents. Note that we use geometric units here in which the velocity of light is equal to 1 . We assume that the matter is such that the following relations hold

$$
\mathbf{H}(\mathbf{x}, \omega)=\mathbf{B}(\mathbf{x}, \omega), \quad \mathbf{D}(\mathbf{x}, \omega)=\epsilon(\mathbf{x}, \omega) \mathbf{E}(\mathbf{x}, \omega) .
$$

In this case the Maxwell equations (4) are equivalent to the vector Helmholtz equation (1).

The permittivity $\epsilon(\mathbf{x}, \omega)$ can depend on $\mathbf{x}$, and it is assumed here that it is piece-wise smooth. This means that it is a smooth function in various domains of $\mathbb{R}^{3}$, but not necessarily continuous on the whole of $\mathbb{R}^{3}$. A typical situation would be that it is constant in a homogeneous compact matter distribution, but may jump at the boundary of the matter which is assumed to be smooth. The matter has to be of finite extension which means that there is vacuum for $\|\mathbf{x}\| \gg 1$, i.e., $\epsilon(\mathbf{x}, \omega)=$ 1 for $\|\mathbf{x}\| \rightarrow \infty$. 
It is well known, see for instance [23] and references therein, that the Maxwell equations in the presence of a symmetry given by a Killing vector (in the axisymmetric case to be considered here this is $\partial_{\phi}$ ) simplify considerably. In this case one can introduce the twist potential, here the $\phi$ component of $\nabla \times \mathbf{E}$. The Maxwell equations are then equivalent to a scalar equation for the twist potential. Whereas our numerical approach does not rely on the presence of such a symmetry, we limit ourselves to this case here since it allows for a more compact presentation of the approach.

In applications, for instance in the description of nano-conductors in optics, see [11] and references therein, the Green's functions of the studied Maxwell equations play an important role. Therefore we mention how the latter are related to the equations we actually solve.

2.1. Spherical coordinates. We first consider spherical coordinates,

$$
x_{1}=r \cos \phi \sin \theta, \quad x_{2}=r \sin \phi \sin \theta, \quad x_{3}=r \cos \theta,
$$

where $r \in \mathbb{R}^{+}, \theta \in[0, \pi]$ and $\phi \in[0,2 \pi[$. The electric field has the components $E^{r}, E^{\theta}$ and $E^{\phi}$ in these coordinates.

We concentrate here on the axisymmetric case where $E^{r}, E^{\theta}$ depend only on $r, \theta$ and $\omega$, and where $E^{\phi}(r, \theta, \omega)=0$. Let $G$ be the twist potential, with [26],

$$
G=\frac{1}{r}\left(\left(r E^{\theta}\right)_{r}-E_{\theta}^{r}\right) .
$$

For the Helmholtz equation (11) we get with [26] and (76)

$$
\begin{aligned}
(G \sin \theta)_{\theta} \frac{1}{\sin \theta}-\omega^{2} \epsilon r E^{r} & =r f^{r}, \\
(r G)_{r}+\omega^{2} \epsilon r E^{\theta} & =-r f^{\theta} .
\end{aligned}
$$

This system, together with some boundary conditions to be detailed in the following section, determines $E^{r}$ and $E^{\theta}$.

Putting $Y=G \sin \theta$ and $x=\cos \theta$, system (8) is equivalent to

$$
\begin{aligned}
Y_{x}+\omega^{2} \epsilon r E^{r} & =-r f^{r}, \\
(r Y)_{r}+\omega^{2} \epsilon r \sin \theta E^{\theta} & =-r \sin \theta f^{\theta} .
\end{aligned}
$$

By differentiating (9), we get for constant $\epsilon$

$$
r^{2}\left(Y_{r r}+\omega^{2} \epsilon Y\right)+2 r Y_{r}+\left(1-x^{2}\right) Y_{x x}=f,
$$

where $f=r \sin \theta\left(-\left(r f^{\theta}\right)_{r}+f_{g}^{r}\right)_{2}$ For given $Y$, the components $E^{r}$ and $E^{\theta}$ can be obtained from $(9)$ for non-vanishing $\epsilon$ and $\omega$. Thus in the axisymmetric case, the solution of the vector Helmholtz equation (11) is equivalent to the solution of the scalar Helmholtz-type equation (10). Note that this equation is singular for $r=0$ and $r \rightarrow \infty$, and for $x= \pm 1$, the axis of symmetry. 
Equation (10) can be separated (we only consider the homogeneous equation here) and solved in terms of spherical Bessel functions $Z_{l}(y)$, i.e., solutions to

$$
y^{2} Z_{l}^{\prime \prime}(y)+2 y Z_{l}^{\prime}(y)+\left(y^{2}-l(l+1)\right) Z_{l}(y)=0,
$$

and derivatives of Legendre polynomials $P_{l}(x), l \in \mathbb{N}$, see [1]. The general formal solution of the homogeneous equation can thus be written in the form

$$
Y=\left(1-x^{2}\right) \sum_{l=1}^{\infty} a_{l} P_{l}^{\prime}(x) Z_{l}(k r)
$$

where $a_{l}, l \in \mathbb{N}$ are constants, and where $k=\omega \sqrt{\epsilon}$. The functions $Z_{l}(k r)$, also called cylinder functions are linear combinations of the spherical Bessel or Neumann functions, or of the Hankel functions, see [1]. Near the origin, only the Bessel functions are regular, near infinity the Sommerfeld condition determines the corresponding Hankel functions.

If one is interested in the solution of equation $(10)$ for arbitrary $f$, it might be useful to introduce the scalar Green's function $g$ such that

$$
Y=\mathbf{g} \otimes f
$$

where $\otimes$ denotes the convolution in $x$ and $r$. Formally $\mathrm{g}$ can be obtained by solving 10 with $f=\delta^{(2)}$ where $\delta^{(2)}$ is the two-dimensional delta-function. In a similar way one can define the Green's function of the system (9),

$$
\left(\begin{array}{l}
E^{r} \\
E^{\theta}
\end{array}\right)=\left(\begin{array}{ll}
\mathcal{G}^{r r} & \mathcal{G}^{r x} \\
\mathcal{G}^{x r} & \mathcal{G}^{x x}
\end{array}\right) \otimes\left(\begin{array}{l}
f^{r} \\
f^{\theta}
\end{array}\right)
$$

The entries of the Green's function in 13 follow for non-vanishing $\omega$ and $\epsilon$ for given $\mathcal{G}$ from $(9)$,

$$
\begin{aligned}
\omega^{2} \epsilon \mathcal{G}^{r r} & =-\delta^{(2)}+\frac{1}{r} \mathbf{g}_{x} \otimes\left[r\left(1-x^{2}\right) \partial_{x}\right], \\
\omega^{2} \epsilon \mathcal{G}^{r x} & =\frac{1}{r} \mathbf{g}_{x} \otimes\left[r \sqrt{1-x^{2}} \partial_{r} r\right], \\
\omega^{2} \epsilon \mathcal{G}^{x r} & =\frac{1}{r \sqrt{1-x^{2}}}(r \mathbf{g})_{r} \otimes\left[r\left(1-x^{2}\right) \partial_{x}\right], \\
\omega^{2} \epsilon \mathcal{G}^{x x} & =-\delta^{(2)}+\frac{1}{r \sqrt{1-x^{2}}}(r \mathbf{g})_{r} \otimes\left[r \sqrt{1-x^{2}} \partial_{r} r\right] .
\end{aligned}
$$

2.2. Prolate spheroidal coordinates. Prolate spheroidal coordinates $\eta, \theta$ and $\phi$ with $0 \leq \eta<\infty, 0 \leq \theta<\pi$ and $0 \leq \phi<2 \pi$ are related to 
Cartesian coordinates via

$$
\begin{aligned}
& x_{1}=a \sinh \eta \sin \theta \cos \phi, \\
& x_{2}=a \sinh \eta \sin \theta \sin \phi, \\
& x_{3}=a \cosh \eta \cos \theta .
\end{aligned}
$$

Constant coordinate surfaces are

$$
\frac{x_{1}^{2}+x_{2}^{2}}{a^{2} \sinh ^{2} \eta}+\frac{x_{3}^{2}}{a^{2} \cosh ^{2} \eta}=1,
$$

and

$$
\frac{x_{1}^{2}+x_{2}^{2}}{a^{2} \sin ^{2} \theta}-\frac{x_{3}^{2}}{a^{2} \cos ^{2} \theta}=-1 .
$$

We show examples of these constant coordinate surfaces in Fig. 11 in the $\varrho, x_{3}$ plane where $\varrho:=\sqrt{x_{1}^{2}+x_{2}^{2}}$ in Fig. 1 (obviously there is a rotational symmetry with respect to the $\varrho=0$ axis). The spheroids can be seen on the left of the figure, the paraboloids on the right. The
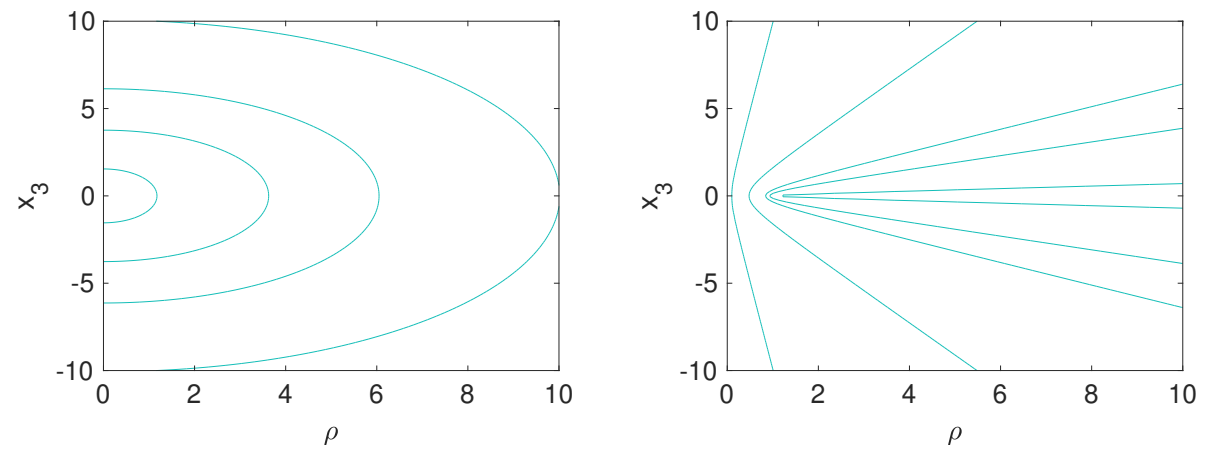

Figure 1. Constant coordinate contours in the $\varrho, x_{3^{-}}$ plane, on the left (16) for $\eta=1,2,2.5,3$ (from left to right), and on the right (17) for $\theta=0.1,0.5,1,1.2,1.5$ (also from left to right).

reader is referred to [26] for more information on these coordinates.

As in the case of spherical coordinates, we concentrate on a situation with axial symmetry, i.e., $E^{\phi}=0$, and no dependence of $\mathbf{E}$ on the coordinate $\phi$. We introduce again the twist potential

$$
F:=(\nabla \times \mathbf{E})_{\phi}=\frac{1}{a \Psi}\left(\left(\sqrt{\Psi} E^{\theta}\right)_{\eta}-\left(\sqrt{\Psi} E^{\eta}\right)_{\theta}\right),
$$

where we have put $\Psi_{\mathrm{s}}=\sinh ^{2} \eta+\sin ^{2} \theta$.

This implies with [26] for the vector Helmholtz equation (1)

$$
\begin{gathered}
(F \sin \theta)_{\theta} \frac{1}{\sin \theta}-\omega^{2} \epsilon a \sqrt{\Psi} E^{\eta}=a \sqrt{\Psi} f^{\eta}, \\
(F \sinh \eta)_{\eta} \frac{1}{\sinh \eta}+\omega^{2} \epsilon a \sqrt{\Psi} E^{\theta}=-a \sqrt{\Psi} f^{\theta} .
\end{gathered}
$$


The functions $E^{\eta}$ and $E^{\theta}$ can be determined from this system.

We put $x=\cos \theta$ and $y=\cosh \eta$, which implies $\Psi=y^{2}-x^{2}$, and

$$
X:=\sin \theta \sinh \eta F .
$$

The system (19) then takes the form

$$
\begin{aligned}
X_{x}+\omega^{2} \epsilon a \sqrt{\psi} \sinh \eta E^{\eta} & =-a \sqrt{\psi} \sinh \eta f^{\eta}, \\
X_{y}+\omega^{2} \epsilon a \sqrt{\psi} \sin \theta E^{\theta} & =-a \sqrt{\psi} \sin \theta f^{\theta} .
\end{aligned}
$$

By differentiating, the system (21) is equivalent for constant $\epsilon$ to a scalar Helmholtz-type equation,

$$
\left(y^{2}-1\right)\left(X_{y y}+\omega^{2} \epsilon a^{2} X\right)+\left(1-x^{2}\right)\left(X_{x x}+\omega^{2} \epsilon a^{2} X\right)=g,
$$

where

$$
g=-a \sqrt{\left(1-x^{2}\right)\left(y^{2}-1\right)}\left[\sqrt{y^{2}-1}\left(f^{\theta} \sqrt{\Psi}\right)_{y}+\sqrt{1-x^{2}}\left(f^{\eta} \sqrt{\Psi}\right)_{x}\right] .
$$

The homogeneous equation (22) can be separated in the form

$$
X(y, x)=\sqrt{\left(1-x^{2}\right)\left(y^{2}-1\right)} R_{\kappa}(y) S_{\kappa}(x),
$$

where $\kappa$ is a complex constant, which leads to the ODEs

$$
\left(y^{2}-1\right) R_{\kappa}^{\prime \prime}(y)+2 y R_{\kappa}^{\prime}(y)+\left(\omega^{2} \epsilon a^{2}\left(y^{2}-1\right)-\kappa-\frac{1}{y^{2}-1}\right) R_{\kappa}(y)=0,
$$

$$
\left(1-x^{2}\right) S_{\kappa}^{\prime \prime}(x)-2 x S_{\kappa}^{\prime}(x)+\left(\omega^{2} \epsilon a^{2}\left(1-x^{2}\right)+\kappa-\frac{1}{1-x^{2}}\right) S_{\kappa}(x)=0 .
$$

Its solutions are known as prolate spheroidal wave functions, see for instance [1]. The eigenvalues $\kappa$ are determined by the regularity of the solutions to the second equation on the axis $(x= \pm 1)$. Thus the solution of $(22)$ can be formally written in the form

$$
X=\sqrt{\left(1-x^{2}\right)\left(y^{2}-1\right)} \sum_{\kappa \in K} R_{\kappa}(y) S_{\kappa}(x),
$$

where $K$ denotes the (infinite) discrete spectrum of $(23)$.

If $\omega$ and $\epsilon$ do not vanish, $E^{\eta}$ and $E^{\theta}$ can be obtained via 20 for given $X$. Thus the scalar Helmholtz-type equation (22) is in this case equivalent to the Helmholtz equation (11). Equation (22) is singular for $y=1$ (the origin) and $y \rightarrow \infty$, and on the axis $x= \pm 1$.

As in the case of spherical coordinates in the previous subsection, it can be useful to introduce the Green's function $g$ such that

$$
X=\mathbf{g} \otimes g
$$

where $\otimes$ denotes now the convolution in $x$ and $y$. The function $\mathbf{g}$ is the solution of $(22)$ with $g=\delta^{(2)}$ where $\delta^{(2)}$ is now the two-dimensional 
delta-function depending on $x$ and $y$. The Green's function of the system (21) can be written in the form

Greenps2 (26)

$$
\left(\begin{array}{l}
E^{\eta} \\
E^{\theta}
\end{array}\right)=\left(\begin{array}{ll}
\mathcal{G}^{y y} & \mathcal{G}^{y x} \\
\mathcal{G}^{x y} & \mathcal{G}^{x x}
\end{array}\right)=\left(\begin{array}{l}
f^{\eta} \\
f^{\theta}
\end{array}\right)
$$

For non-vanishing $\omega$ and $\epsilon$ one gets for the Green's function in $(26)$

$$
\begin{aligned}
\omega^{2} \epsilon \mathcal{G}^{y y} & =-\sqrt{y^{2}-1} \delta^{(2)}+\frac{1}{\sqrt{\psi}} \mathbf{g}_{x} \otimes\left[\left(y^{2}-1\right) \sqrt{1-x^{2}} \partial_{x} \sqrt{\psi}\right] \\
\omega^{2} \epsilon \mathcal{G}^{y x} & =\frac{1}{\sqrt{\psi}} \mathbf{g}_{x} \otimes\left[\left(1-x^{2}\right) \sqrt{y^{2}-1} \partial_{y} \sqrt{\psi}\right] \\
\omega^{2} \epsilon \mathcal{G}^{x y} & =\frac{1}{\sqrt{\psi}} \mathbf{g}_{y} \otimes\left[\left(y^{2}-1\right) \sqrt{1-x^{2}} \partial_{x} \sqrt{\psi}\right], \\
\omega^{2} \epsilon \mathcal{G}^{x x} & =-\sqrt{1-x^{2}} \delta^{(2)}-\frac{1}{\sqrt{\psi}} \mathbf{g}_{y} \otimes\left[\left(1-x^{2}\right) \sqrt{y^{2}-1} \partial_{y} \sqrt{\psi}\right] .
\end{aligned}
$$

\section{Sommerfeld AND MATChing CONDitions}

In this section we summarize the matching conditions at the domain boundaries, and how the Sommerfeld radiation condition at infinity can be implemented.

Since the Helmholtz equation is a second order PDE, one needs two matching conditions at each domain in order to obtain a unique solution. Since the domains are chosen in way that their boundary is a constant surface in the 'radial' coordinate, this means that a condition on the function and its normal derivative are required at each domain boundary. In domains where the equations are singular, the number of conditions can be less as detailed below.

3.1. Sommerfeld radiation condition. The Sommerfeld condition (2) ensures that there is no incoming radiation from infinity. It implies that the solutions of the Helmholtz equation (1) can be written in the form

$$
\mathbf{E}=e^{-i \omega|| \mathbf{x} \|} \tilde{\mathbf{E}}(\|\mathbf{x}\|, \omega),
$$

where $\tilde{\mathbf{E}}$ is a non-oscillatory function vanishing for $\|\mathbf{x}\| \rightarrow \infty$. Note that we assume that $\epsilon(\|\mathbf{x}\|, \omega)=1$ in an open environment of $\infty$, i.e., that all matter in the studied models is of finite volume.

In spherical coordinates this implies that we can introduce in the vicinity of infinity the local parameter $\rho=1 / r$ and split off the oscillatory terms as in 28),

$$
E^{r}=e^{-i \omega r} \tilde{E}^{r}, \quad E^{\theta}=e^{-i \omega r} \tilde{E}^{\theta}, \quad Y=e^{-i \omega r} \tilde{Y} .
$$

Thus we get for equation 10 near infinity

$$
\rho^{2} \tilde{Y}_{\rho \rho}+2 i \omega \tilde{Y}_{\rho}-\frac{2 i \omega}{\rho} \tilde{Y}+\left(1-x^{2}\right) \tilde{Y}_{x x}=e^{i \omega / \rho} f .
$$


Note that this equation is singular for $\rho=0$ and $x= \pm 1$, i.e., at infinity and on the symmetry axis.

In the prolate spheroidal coordinates, we make with $(28)$ the ansatz

$$
E^{\eta}=e^{-i \omega a y} \tilde{E}^{\eta}, \quad E^{\theta}=e^{-i \omega a y} \tilde{E}^{\theta}, \quad X=e^{-i \omega a y} \tilde{X}
$$

and introduce the local variable $\xi=1 / y$ near infinity. Thus we get for (22)

(32) $\left(1-\xi^{2}\right)\left(\xi^{2} \tilde{X}_{\xi \xi}+2(\xi-i \omega a) \tilde{X}_{\xi}\right)+\left(1-x^{2}\right)\left(\tilde{X}_{x x}+\omega^{2} a^{2} \tilde{X}\right)=e^{i \omega a / \xi} g$.

Note that this equation is singular for $\xi=0$ and $x= \pm 1$, i.e., at infinity and on the symmetry axis.

3.2. Matching conditions. The spectral methods we intend to apply in this paper are especially efficient if the physical boundaries coincide with domain boundaries, i.e., with constant coordinate surfaces. Therefore we discuss in this paper two sets of spheroidal coordinates and assume that $\epsilon$ is smooth or constant except for a finite number of values for the coordinate $r$ and $\eta$ respectively.

It is known that in the absence of surface densities and currents, the normal components of $\mathbf{D}$ and $\mathbf{B}$ are continuous at the boundary, and that the same holds for the tangential components of $\mathbf{E}$ and $\mathbf{H}$. This means that in our cases $E^{\theta}$ is continuous as well as $\epsilon E^{r}$ or $\epsilon E^{\eta}$ in spherical or prolate spheroidal coordinates respectively.

The matching conditions for the function $Y$ can be read off from $(8)$ : $Y$ and $Y_{m} / \epsilon$ are continuous at the boundaries. In a similar way the system (19) gives the matching conditions for the function $X: X$ as well as $X_{\eta} / \epsilon$ are continuous at the boundaries.

We will work with $N_{d}+1$ domains which are defined by the constant radii $r^{(i)} \leq r^{(i+1)}, i=1, \ldots, N_{d}$, in spherical coordinates and the constants $y^{(i)} \leq y^{(i+1)}, i=1, \ldots, N_{d}$, in prolate spheroidal coordinates: $i=1: r<r^{(1)}\left(y<y^{(1)}\right)$ : Near the origin, a singularity of the equations, special conditions need to be imposed to ensure a regular solution. In the spherical case, the function $Y$ vanishes for $r=0$, since the spherical Bessel functions in (11) vanish there for $l>0$ :

$$
Y^{(1)}(0, x, \omega)=0 .
$$

In the prolate spheroidal case, $X$ must vanish at the origin in order to have a regular solution. In addition we impose that the functions $Y$, $X$ are continuous,

$$
Y^{(1)}\left(r^{(1)}, x, \omega\right)=Y^{(2)}\left(r^{(1)}, x, \omega\right),
$$

where $Y^{(1)}$ is the function in domain I, and $Y^{(2)}$ is the function in domain II. Similarly we have

$$
X^{(1)}\left(y^{(1)}, x, \omega\right)=X^{(2)}\left(y^{(1)}, x, \omega\right) .
$$

$1<i<N_{d}-1: r^{(i-1)}<r<r^{(i)}\left(y^{(i-1)}<y<y^{(i)}\right)$ : here we have to impose two conditions since there is no singularity in the radial 
coordinates. We impose continuity of the respective function at $r^{(i)}$, and a condition on the radial derivative at $r^{(i-1)}$ :

$$
\begin{aligned}
Y^{(i)}\left(r^{(i)}, x, \omega\right) & =Y^{(i+1)}\left(r^{(i)}, x, \omega\right), \\
\frac{Y_{r}^{(i-1)}\left(r^{(i-1)}, x, \omega\right)}{\epsilon^{(i-1)}\left(r^{(i-1)}, \omega\right)} & =\frac{Y_{r}^{(i)}\left(r^{(i-1)}, x, \omega\right)}{\epsilon^{(i)}\left(r^{(i-1)}, \omega\right)},
\end{aligned}
$$

where $\epsilon^{(i)}$ are the values of $\epsilon$ in the respective domain.

In prolate spheroidal coordinates we have the matching conditions

$$
\begin{aligned}
X^{(i)}\left(y^{(i)}, x, \omega\right) & =X^{(i+1)}\left(y^{(i)}, x, \omega\right), \\
\frac{X_{y}^{(i-1)}\left(y^{(i-1)}, x, \omega\right)}{\epsilon^{(i-1)}\left(y^{(i-1)}, \omega\right)} & =\frac{X_{y}^{(i)}\left(y^{(i-1)}, x, \omega\right)}{\epsilon^{(i)}\left(y^{(i-1)}, \omega\right)} .
\end{aligned}
$$

$i=N_{d}$ :

In the domain bordering to the infinite one, the above conditions change as follows

$$
\begin{aligned}
Y^{\left(N_{d}\right)}\left(r^{\left(N_{d}\right)}, x, \omega\right) & =e^{-i \omega r^{\left(N_{d}\right)}} \tilde{Y}\left(1 / r^{\left(N_{d}\right)}, x, \omega\right), \\
\frac{Y_{r}^{\left(N_{d}-1\right)}\left(r^{\left(N_{d}-1\right)}, x, \omega\right)}{\epsilon^{\left(N_{d}-1\right)}\left(r^{\left(N_{d}-1\right)}, \omega\right)} & =\frac{Y_{r}^{N_{d}}\left(r^{\left(N_{d}-1\right)}, x, \omega\right)}{\epsilon^{\left(N_{d}-1\right)}\left(r^{\left(N_{d}-1\right)}, \omega\right)},
\end{aligned}
$$

and

$$
\begin{aligned}
X^{\left(N_{d}\right)}\left(y^{\left(N_{d}\right)}, x, \omega\right) & =e^{-i \omega a y^{\left(N_{d}\right)}} \tilde{X}\left(1 / y^{\left(N_{d}\right)}, x, \omega\right), \\
\frac{X_{y}^{\left(N_{d}-1\right)}\left(y^{\left(N_{d}-1\right)}, x, \omega\right)}{\epsilon^{\left(N_{d}-1\right)}\left(y^{\left(N_{d}-1\right)}, \omega\right)} & =\frac{X_{y}^{\left(N_{d}\right)}\left(y^{\left(N_{d}-1\right)}, x, \omega\right)}{\epsilon^{\left(N_{d}\right)}\left(y^{\left(N_{d}-1\right)}, \omega\right)},
\end{aligned}
$$

$r>r^{\left(N_{d}\right)}\left(y>y^{\left(N_{d}\right)}\right)$ : infinity is a singularity of the equations and (32), but the vanishing of the respective solution at infinity has to be imposed in spherical coordinates (no condition is needed in the prolate spheroidal case). In addition we impose the matching condition on the radial derivative,

YIII (40)

$$
\frac{Y_{r}^{\left(N_{d}\right)}\left(r^{\left(N_{d}\right)}, x, \omega\right)}{\epsilon^{\left(N_{d}\right)}\left(r^{\left(N_{d}\right)}, \omega\right)}=e^{-i \omega r^{\left(N_{d}\right)}}\left(\tilde{Y}_{\rho}\left(1 / r^{\left(N_{d}\right)}, x, \omega\right)-i \omega \tilde{Y}\left(1 / r^{\left(N_{d}\right)}, x, \omega\right)\right) .
$$

In prolate spheroidal coordinates we have

$$
\frac{X_{y}^{\left(N_{d}\right)}\left(y^{\left(N_{d}\right)}, x, \omega\right)}{\epsilon^{\left(N_{d}\right)}\left(y^{\left(N_{d}\right)}, \omega\right)}=e^{-i \omega a y^{\left(N_{d}\right)}}\left(\tilde{X}_{\xi}\left(1 / y^{\left(N_{d}\right)}, x, \omega\right)-i \omega a \tilde{X}\left(1 /^{\left(N_{d}\right)}, x, \omega\right)\right) \text {. }
$$

\section{NumericAl APPROACH}

In this section we briefly describe the numerical approach for the Helmholtz equations. In the angular coordinate, we always use a Chebyshev collocation method, in the 'radial' coordinate, we consider several domains such that the line $\mathbb{R}^{+}$is completely covered by these domains (infinity is simply a point on the grid). On each domain we use 
once more a Chebyshev collocation method. The matching conditions are imposed via a $\tau$-method 25 .

The essence of spectral methods is to approximate functions on a finite interval via functions being globally smooth on the considered interval. It is known that analytical functions are approximated by spectral methods with an error decreasing exponentially with the number of collocation points. Here we apply a Chebyshev collocation method, see [30] for details: the function to be approximated is sampled on the Chebyshev points $l_{n}=\cos (n \pi / N), n=0, .1, \ldots, N$ with $N \in \mathbb{N}$. A function $u(l)$ is approximated on the interval $[-1,1]$ by the Lagrange polynomial $p(l)$ of degree $N$ passing through the collocation points, $p\left(l_{n}\right)=u\left(l_{n}\right), n=0, \ldots, N$. The derivative of $u$ with respect to the argument is approximated via the derivative of the Lagrange polynomial which leads to the action of a Chebyshev differentiation matrix $D$ on the vector $\mathrm{u}$ with components $u\left(l_{0}\right), \ldots, u\left(l_{N}\right)$, i.e., $u^{\prime} \approx D \mathrm{u}$. These Chebyshev differentiation matrices can be found for instance in [30, 33].

For the angular variable $x$, this method can be applied as described above since $x \in[-1,1]$. In the radial coordinate ( $r$ or $y$ in the spherical and prolate spheroidal coordinates respectively), we introduce a number of domains which are chosen such that $\epsilon$ is smooth on each domain. Thus there will be a collection of radii $r^{(i)}<r^{(i+1)}$ (we only describe in the following this case since the treatment in $y$ is analogous), $i=$ $1, \ldots, N_{d}$. Domain I is given by $r \leq r^{(1)}$ and thus contains the origin, domain $N_{d}+1$ is defined via $r>r^{\left(N_{d}\right)}$ is infinite and will be compactified. Each of the intervals $\left[r^{(i)}, r^{(i+1)}\right], i=0, \ldots, N_{d}\left(r_{0}=0\right)$ is mapped to the interval $[-1,1]$ via $r=(1+l) / 2 r^{(i+1)}+(1-l) r^{(i)}, l \in[-1,1]$. On the infinite interval, we apply the mapping $r=2 / r_{N_{d}} /(1+l)$. On each interval the standard Chebyshev collocation points are introduced as well as the Chebyshev differentiation matrices. Since the domains II to $N_{d}$ are all identical from a mathematical point of view, we discuss in the following only the case of three domains. A generalization to a larger number $N_{d}>2$ is straight forward.

This approach, i.e., discretization in both $r$ and $x$, allows to approximate the equations (10) and (22) via a system of ordinary differential equations (ODEs) (this is a standard tensor grid). In the infinite domain, we discretize the equations (30) respectively (32). The matching conditions of the previous section are imposed via a $\tau$-method. This means that the equations in each domain corresponding to the radii $r_{i}$ and $r_{i+1}$ are replaced by the matching conditions in subsection 3.2 . For a given right hand side $f$ (discretized in the same way), this leads to an equation of the Form $A Y=f$ for some invertible matrix $A$ after discretization of the differentiation operators. This gives the wanted solution in each domain after solving the resulting linear system. This will be done in Matlab with the command 'backslash', i.e., with essentially Gaussian elimination. If one is interested in the Green's function, 
one simply has to replace the delta function $\delta^{(2)}$ by the identity in the considered vector space.

Note that though the solution is only constructed on the collocation points it can be obtained at all points in $\mathbb{R}^{2}$, with prescribed precision, via interpolation. An efficient and numerically stable way to do this is via barycentric interpolation, see [7] and references therein.

A Chebyshev collocation method as presented above is equivalent to an expansion of a function $u(l)$ in terms of Chebyshev polynomials $T_{n}(l), n \in \mathbb{N}$, where $T_{n}(l)=\cos (n \arccos (l))$. This means one approximates $u$ via

$$
u(l) \approx \sum_{n=0}^{N} a_{n} T_{n}(l) .
$$

The Chebyshev coefficients $a_{n}$ are determined via

$$
u\left(l_{n}\right)=\sum_{m=0}^{N} a_{m} T_{m}\left(l_{n}\right), \quad n=0, \ldots, N .
$$

This corresponds to a Fast Cosine Transform which is related to the fast Fourier transform, see [30], and is thus a very efficient way to compute the coefficients at a computational cost of $\mathcal{O}(N \ln N)$ operations.

The decrease of the spectral coefficients for a smooth function in both coordinates is expected to be exponential. This allows to allocate the numerical resolution in an efficient way. We first choose the domain boundaries according to the physical situation, i.e., discontinuities in the permittivity $\epsilon$ will be located at constant coordinate surfaces by assumption, and these will be chosen to be domain boundaries. Independently of this there will be always one domain containing the origin and one in the vicinity of infinity, both singularities of our equations. There will be always at least one domain in between these two domains, so the minimal number of domains will be three.

In each of these domains, we choose the resolution such that the spectral coefficients decrease to the aimed at accuracy, here essentially machine precision. Since the condition of the $N \times N$ Chebyshev differentiation matrix $D^{2}$ is of order $\mathcal{O}\left(N^{4}\right)$, see [30], one should aim in each domain at a small number of points which will not only lead to better conditioned matrices, but also to faster codes since the total differentiation matrix for all domains has a block structure, except for the matching conditions.

In general one can do an exploratory low resolution run to estimate the optimal number of domains, the location of their boundaries and the resolution in each them. Since spectral methods are efficient for situations with simple geometry, this can be done by hand (this is always possible in our examples). If one were interested in an adaptive approach, one would as in [9] check the three (to avoid that coefficients 
vanish for symmetry reasons) spectral coefficients with the highest index for each domain and for each coordinate, and then vary the resolution until the largest of them in modulus is smaller than the aimed at accuracy.

\section{EXAMPLES}

In this section we study the performance of the presented codes for examples showing typical features of solutions to the Helmholtz equation (1). To construct examples in spherical and prolate spheroidal coordinates, we use what is jokingly called Synge's method in a general relativistic context: we make an ansatz for the solution and compute the right hand side of (11). This gives obviously an exact solution to the equation with this specific right hand side, which is then to be reproduced. Note that the goal of the first two examples is to provide interesting explicit test cases for the codes, not necessarily to study physically interesting situations for which no exact solutions are known. For simplicity we consider for these examples only vacuum, i.e., $\epsilon=1$ everywhere. An application to a typical problem in nano-optics is studied in the last subsection, the interaction of a radiating dipole with a spherical nano-particle, here silver, see e.g. [11] and references therein. In the nano-particle, the permittivity is considered to be given by a Drude model. No exact solution is known in this case with a piecewise constant permittivity, but we are able to numerically resolve this situation in a way that the spectral coefficients decrease to machine precision which can be seen as indicating the numerical accuracy. Note that the radii are chosen in all examples as in the physical problem for convenience.

The codes we apply here are written in Matlab which is an interpreter language. Thus Matlab timings can depend strongly on the way of coding and how much precompiled code is actually used. Therefore the timings have to be taken with a grain of salt. But for practical applications it is of course useful to know at least the order of magnitude of time a run takes. The example of Fig. 2 takes roughly 2 seconds on a laptop, the example in Fig. 5 and in the last subsection roughly 80 seconds.

5.1. Spherical coordinates. In the case of spherical coordinates, we expect the function $Y$ to vanish for $r=0$ and for $r \gg 1$ to be oscillatory of the form (3) in order to satisfy the Sommerfeld condition.

As an example for a function with this behavior we consider

$$
Y=\frac{r e^{-i \omega r}}{1+r^{2}+x^{2}}
$$


This implies with 30 )

$$
\begin{aligned}
f & =e^{-1 i \omega r}\left(\frac{2 r(1-2 i \omega r)}{1+r^{2}+x^{2}}+\frac{2 r^{3}(2 i \omega r-5)}{\left(1+r^{2}+x^{2}\right)^{2}}+\frac{8 r^{5}}{\left(1+r^{2}+x^{2}\right)^{3}}\right. \\
& \left.-\frac{2 r\left(1-x^{2}\right)}{\left(1+r^{2}+x^{2}\right)^{2}}+\frac{8 r x^{2}\left(1-x^{2}\right)}{\left(1 r^{2}+x^{2}\right)^{3}}\right)
\end{aligned}
$$

The source $f$ does not tend to zero at infinity, but this is not necessarily unphysical since we have $f=r^{2} \sin \theta\left(r f_{r}^{\theta}+f_{\theta}^{r}\right)$. Thus the source, for instance a free charge density, is multiplied by a factor $r^{2}$.

We use the three domains $r \leq 8,8<r<20$ and $r>20$. The real part of the solution is shown in these three domains for $\omega=1$ in the upper row of Fig. 2, For the computation we use $N_{I}=60, N_{I I}=30$, $N_{I I I}=30$ and $N_{x}=50$ Chebyshev polynomials. The Chebyshev coefficients in the respective domains can be seen in the lower row of Fig. 2. It can be seen that they decrease with this choice of the number of collocation points to the order of machine precision. Note that the dependence of the solution 42 on the variable $x$ is less pronounced the larger $r$ is. Thus one would be able to deal with less collocation points in domains II and III also in $x$, but in order to simplify the code, we use the same number of collocation points in $x$ in all domains.
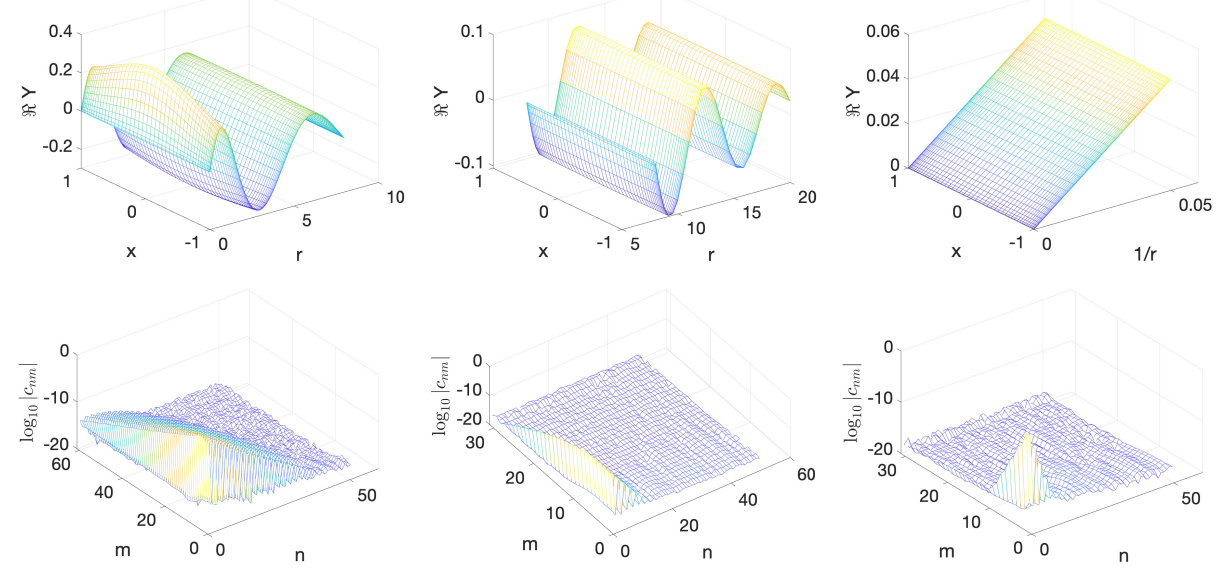

FiguRE 2. Real part of the solution $\sqrt{42}$ for $\omega=1$ in the domains I, II, III (from left to right) in the upper row, and the corresponding Chebyshev coefficients in the lower row.

If we solve equation $(30)$ for the right hand side $(43)$ with the same number of collocation points as in Fig. 2, one gets the difference between exact and numerical solution shown in Fig. 3. It can be seen that it is globally of the order of $10^{-13}$, and thus as expected of the order as indicated by the highest Chebyshev coefficients in the lower row of Fig. 2, 

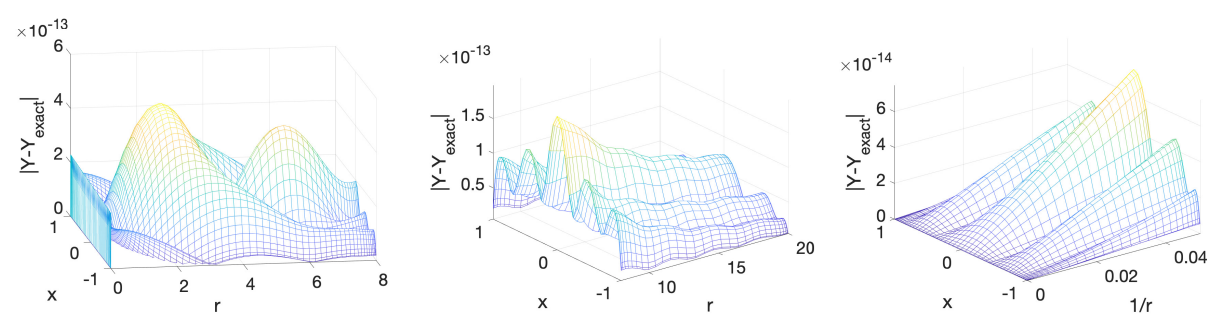

FiguRE 3 Difference of the numerical solution of equation (30) for the right hand side (43) and the exact solution (42) in the domains I, II, III (from left to right).

The dependence of the numerical error on the resolution in $x$ and $r$ can be seen in Fig, 4. For the same values of collocation points in $r$ as in Fig. 2, the dependence of the difference between numerical and exact solution in the $L^{\infty}$ norm in dependence on $N_{x}$ can be seen on the left of Fig. 4. As expected it decreases exponentially and saturates essentially for $N_{x} \geq 30$.
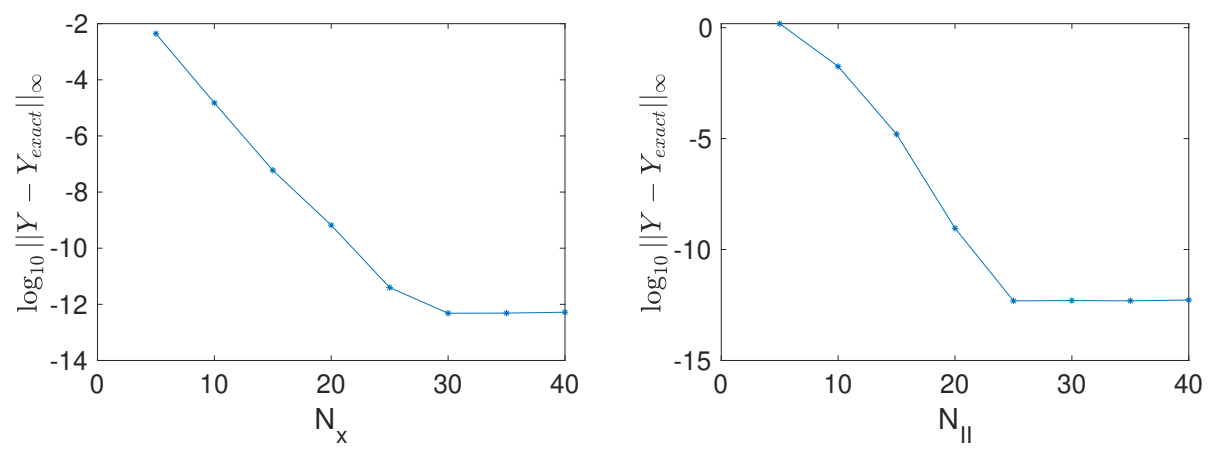

FiguRE 4. $L^{\infty}$ norm of the difference of the numerical solution of equation $(\sqrt{30})$ for the right hand side (43) and the exact solution (42) in dependence of $N_{x}$ on the left, and in dependence of $N_{I I}$ on the right.

Note that though we use three domains in $r$, the solution in these domains are global for $r \in \mathbb{R}^{+}$. This is due to the fact that equation (30) is elliptic, and that we impose on each domain boundary a $C^{1}$ condition on the solution. This leads to an analytical solution for $r \in \mathbb{R}^{+}$. Consequently a lack of resolution in one domain affects the numerical error in all domains. Thus to study the dependence of the numerical error on the resolution in $r$, it is sufficient to compute the global error in dependence of the resolution in just one domain. This error is shown for $N_{x}=50, N_{I}=60$ and $N_{I I I}=30$ in dependence of $N_{I I}$ on the right of Fig. 4. The error decreases as expected exponentially with $N_{I I}$ and saturates for $N_{I I} \sim 25$.

Higher values of $\omega$ lead to a more oscillatory behavior of the solution, see Fig. 5 for $\omega=10$. This will make a higher resolution necessary. 
But with $N_{I}=N_{I I}=100, N_{I I I}=40$ and $N_{x}=100$, we reproduce the solution 42 to the order of $10^{-12}$ in this case.
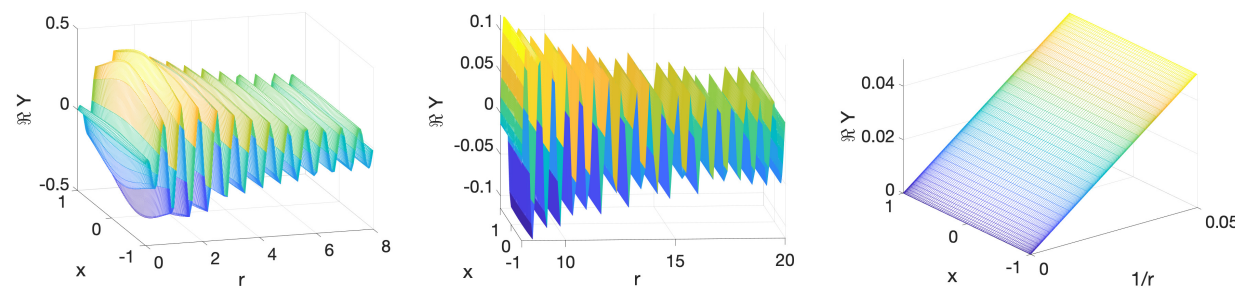

Figure 5. Real part of the solution $\sqrt{42}$ for $\omega=10$ in the domains I, II, III (from left to right).

5.2. Prolate spheroidal coordinates. In the case of prolate spheroidal coordinates, we construct a similar test solution as in the spherical case: the solution must be proportional to $\exp (-i \omega a y) / y$ for $y \rightarrow \infty$, and in addition must vanish for $y=1$. A possible candidate is

$$
X=e^{-i \omega a \sqrt{1+y^{2}}} \frac{y^{2}-1}{\left(y^{2}+x^{2}\right)^{3 / 2}}
$$

With (22) this implies that the source is of the form

$$
\begin{aligned}
g & =\left(1-x^{2}\right)\left(y^{2}-1\right) e^{-i \omega a \sqrt{1+y^{2}}}\left(\frac{3\left(4 x^{2}-y^{2}\right)}{\left(x^{2}+y^{2}\right)^{7 / 2}}+\frac{\omega^{2} a^{2}}{\left(x^{2}+y^{2}\right)^{3 / 2}}\right) \\
& +\left(y^{2}-1\right) e^{-i \omega a \sqrt{1+y^{2}}}\left(\frac{\omega^{2} a^{2}\left(y^{2}-1\right)}{\left(1+y^{2}\right)\left(x^{2}+y^{2}\right)^{3 / 2}}+\frac{2 x^{2}+3-3 y^{2}}{\left(x^{2}+y^{2}\right)^{5 / 2}}-\frac{5 y^{2}\left(2 x^{2}+3-y^{2}\right)}{\left(x^{2}+y^{2}\right)^{7 / 2}}\right. \\
& \left.-\frac{i \omega a y^{2}\left(2 x^{2}+3-y^{2}\right)}{\sqrt{1+y^{2}}\left(x^{2}+y^{2}\right)^{5 / 2}}-\frac{i \omega a\left(3 y^{2}-1\right)}{\sqrt{1+y^{2}}\left(x^{2}+y^{2}\right)^{3 / 2}}+\frac{i \omega a y^{2}\left(y^{2}-1\right)\left(3+x^{2}+4 y^{2}\right)}{\left(1+y^{2}\right)^{3 / 2}\left(x^{2}+y^{2}\right)^{5 / 2}}\right) .
\end{aligned}
$$

We choose the domains with the same values on the axis as before, $a y^{I}=8$ and $a y^{I I}=20$. If the boundary of domain $\mathrm{I}$ in the $x_{3}=0$ plane is $x_{1}=6$, one has $a \sim 5.29$. Solution (44) for these values and $\omega=1$ can be seen in Fig. 6. If we use $N_{I}=N_{I I I}=20$ and $N_{I I}=N_{x}=30$ collocation points, we get the Chebyshev coefficients shown in the lower row of Fig. 6. They decrease in all cases to machine precision.

For this choice of the numerical parameters the difference between numerical and exact solution is shown in Fig. 7. As expected from the Chebyshev coefficients, the error is globally of the order of $10^{-14}$ in this case.

The dependence of the numerical error on the resolution can be again studied by varying the number of collocation points. On the left of Fig. 8, the same number of collocation points in $y$ is applied, and $N_{x}$ varies. It can be seen that the error decreases exponentially and 

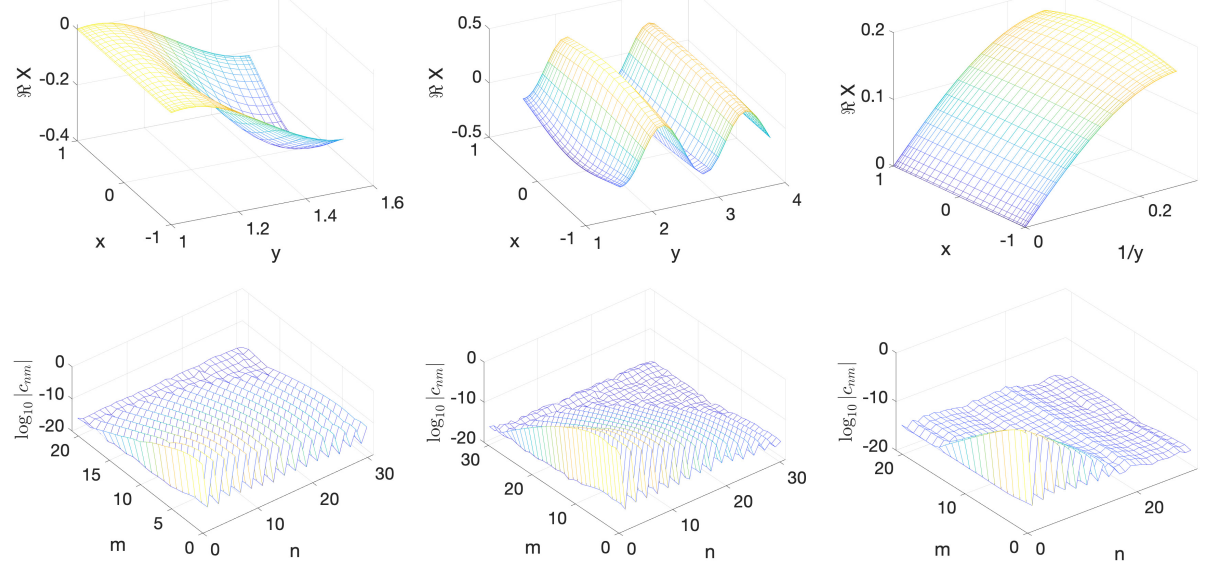

Figure 6. Real part of the solution (44) for $\omega=1$ in the domains I, II, III (from left to right) in the upper row, and the corresponding Chebyshev coefficients in the lower row.
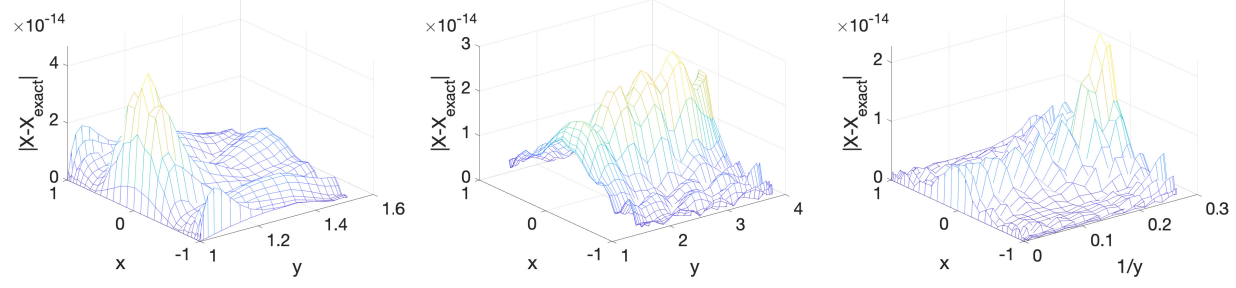

FIgURE 7 Difference of the numerical solution of equation (32) for the right hand side (45) and the exact solution (44) in the domains I, II, III (from left to right).
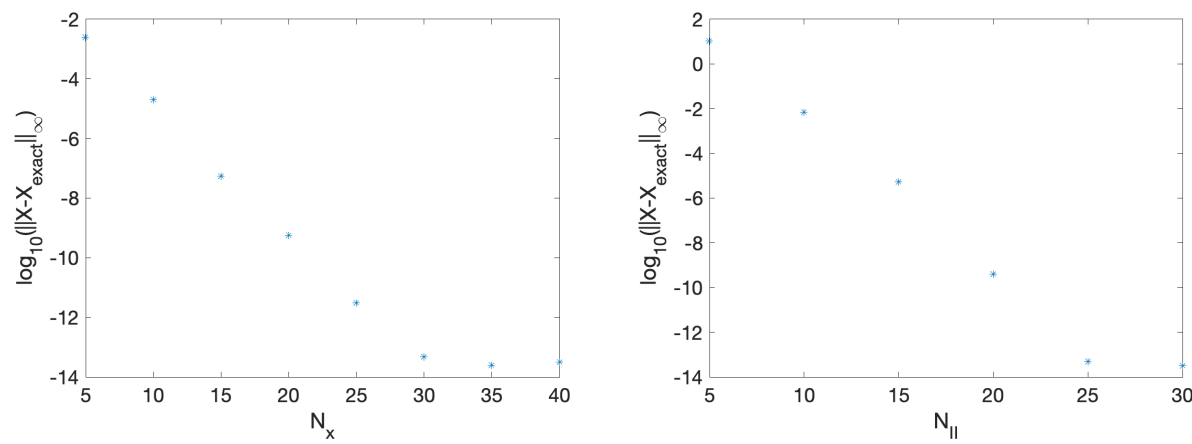

FiguRE $8 . L^{\infty}$ norm of the difference of the numerical solution of equation (32) for the right hand side (45) and the exact solution (44) in dependence of $N_{x}$ on the left, and in dependence of $N_{I I}$ on the right). 
saturates for $N_{x} \sim 30$. On the right of the same figure, $N_{I I}$ is varied. Again the error decreases exponentially and saturates for $N_{I I} \sim 25$.

For larger values of $\omega$, but the same value of $a$, the resolution has to be adjusted since the solution becomes more oscillatory. For $\omega=10$, we use $N_{I}=40, N_{I I}=100, N_{I I I}=30$ and $N_{x}=30$ and reach a global error of the order of $10^{-14}$. If instead we consider $\omega=1$, but change $a$ and thus the shape of the constant coordinate surfaces, no higher resolution is needed. We keep the intersection of the cigar like constant coordinate surface such that it intersects the $x_{3}$-axis for $x_{3}=8$, but change the intersection with the $x_{1}, x_{2}$-plane from 6 to 2

this implies we use a larger $a \sim 7.75$. The situation is shown in Fig. 9 on the left. With the same numerical parameters as in Fig. 6, we get again a global error of the order of $10^{-14}$.
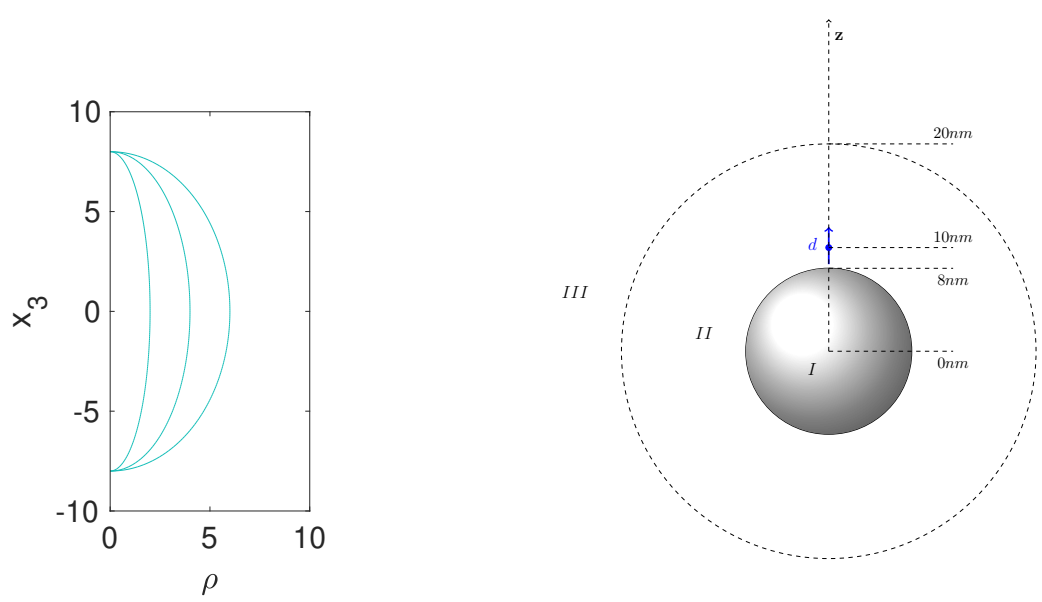

Figure 9. On the left: domain boundaries for 3 cigar shaped domains all hitting the symmetry axis for $x_{3}=$ \pm 8 , but the $\varrho$ axis in the points $2,4,6$ from left to right

; on the right: spherical nano-particle of radius $8 \mathrm{~nm}$ and a dipole on

\subsection{Far field of a strongly coupled dipole-nanoparticle system.}

In this subsection we study the interaction of a spherical nano-particle with a monochromatically radiating dipole. Since we are here interested in a concrete physical problem, the speed of light $c$ is in this subsection considered in SI units.

The dipole can be placed without loss of generality on the $x_{3}$ axis and will be located there at $x_{3}=z_{0}$ outside of the nano-particle, but close to its surface. It is known that the dipole in vacuum leads to the electric 
field $\left(\mathbf{x}_{\mathbf{0}}=z_{0} \hat{x}_{3}\right.$, where $\hat{x}_{3}$ is the unit vector in the $x_{3}$ direction)

$$
\begin{aligned}
\mathbf{E}_{d} & =\left(\frac{k^{2}}{R^{3}}\left(\left(\mathbf{x}-\mathbf{x}_{\mathbf{0}}\right) \times \mathbf{p}\right) \times\left(\mathbf{x}-\mathbf{x}_{\mathbf{0}}\right)\right. \\
& \left.+\left(3\left(\mathbf{x}-\mathbf{x}_{\mathbf{0}}\right)\left(\left(\mathbf{x}-\mathbf{x}_{\mathbf{0}}\right) \mathbf{p}\right)-\mathbf{p} R^{2}\right)\left(\frac{1}{R^{5}}+\frac{i k}{R^{4}}\right)\right) e^{-i k R},
\end{aligned}
$$

where $k=\omega / c$ and where

RzO

$$
R:=\sqrt{r^{2}-2 r x z_{0}+z_{0}^{2}}
$$

The field $\mathbf{E}_{d}$ is the solution to the vector Helmholtz equation (1) in vacuum,

$$
\nabla \times \nabla \times \mathbf{E}_{d}(\mathbf{x}, \omega)-k^{2} \mathbf{E}_{d}(\mathbf{x}, \omega)=\mathbf{S}
$$

where $\mathbf{S}$ is a distributional source. The dipole is chosen to point in the $x_{3}$ direction, $\mathbf{p}=p \hat{x}_{3}$. Thus we get in spherical coordinates

$$
E_{d}^{r}=p e^{-i k R}\left(\frac{k^{2} z_{0} r\left(1-x^{2}\right)}{R^{3}}+\left(3\left(r x-z_{0}\right)\left(r-x z_{0}\right)-x R^{2}\right)\left(\frac{1}{R^{5}}+\frac{i k}{R^{4}}\right)\right)
$$

and

$E_{d}^{\theta}=p \sin \theta e^{-i k R}\left(-\frac{k^{2} r\left(r-x z_{0}\right)}{R^{3}}+\left(3\left(r x-z_{0}\right) z_{0}+R^{2}\right)\left(\frac{1}{R^{5}}+\frac{i k}{R^{4}}\right)\right)$.

Since $Y=\left(\sin \theta\left(r E^{\theta}\right)_{r}+\left(1-x^{2}\right) E_{x}^{r}\right) / r$, we get for the twist potential $Y_{d}$ of the dipole

$$
Y_{d}=p\left(1-x^{2}\right) e^{-i k R}\left(\frac{i k^{3} r}{R^{2}}+\frac{k^{2} r}{R^{3}}\right) .
$$

The radiating dipole to interacts with the spherical metallic nanoparticle. The question is how this nano-particle acts as a resonator. We consider a typical situation of strong interaction [11], a silver particle of radius of $8 \mathrm{~nm}$, the dipole coupled to it is placed $2 \mathrm{~nm}$ from the north pole on the $x_{3}$-axis, thus $z_{0}=10 \mathrm{~nm}$, see Fig. 9 on the right. The permittivity of the nano-particle is modeled by Drude's formula

$$
\epsilon^{I}=\epsilon_{\infty}-\frac{\omega_{p}^{2}}{\omega^{2}+\mathrm{i} \gamma_{p} \omega} ;
$$

for silver [36], the appropriate values are $\epsilon_{\infty}=6, \hbar \omega_{p}=7.90 \mathrm{eV}$, and $\hbar \gamma_{p}=51 \mathrm{meV}$.

Since the dipole is singular at $\mathbf{x}_{0}$ and since $\mathbf{S}$ corresponds to a delta function, the problem is not suited for a direct numerical treatment without taking care of the singularities. However, we know the dipole field in vacuum, and we are only interested in the radiation it causes. Since the Maxwell equations and the Helmholtz equation (1) are linear, 
we can make the ansatz $\mathbf{E}=\mathcal{E}+\mathbf{E}_{d}$. With (1) and (48) we get that the radiation field $\mathcal{E}$ satisfies the equation

$$
\nabla \times \nabla \times \mathcal{E}(\mathbf{x}, \omega)-\omega^{2} \epsilon(\mathbf{r}, \omega) \mathcal{E}(\mathbf{x}, \omega)=\omega^{2}(\epsilon(\mathbf{r}, \omega)-1) \mathbf{E}_{d} .
$$

This means that the dipole field acts as a source for the field $\mathcal{E}$, but just where $\epsilon(\mathbf{r}, \omega) \neq 1$, in our case the interior of the nano-particle. Since the dipole is located outside of the nano-particle, the quantity $R$ will not vanish there. If we introduce the twist potential $\mathcal{Y}$ for $\mathcal{E}$, it satisfies with

$$
r^{2}\left(\mathcal{Y}_{r r}+\omega^{2} \epsilon \mathcal{Y}\right)+2 r \mathcal{Y}_{r}+\left(1-x^{2}\right) \mathcal{Y}_{x x}=f,
$$

where

$$
f=\omega^{2}(\epsilon-1) r \sin \theta\left(\left(-r E_{d}^{\theta}\right)_{r}+\left(E_{d}^{r}\right)_{x}\right)=-\omega^{2}(\epsilon-1) r^{2} Y_{d}
$$

Thus the source $f$ vanishes for $r \rightarrow 0$. We normalize the solution in the following at infinity such that in the $x_{3}=0$ plane we have

$$
\lim _{r \rightarrow \infty} \frac{\mathcal{Y}}{Y_{d}}=1
$$

In other words we divide $\mathcal{Y}$ by $i k^{3} p$.

The resolution for the computation of $\mathcal{Y}$ can be estimated from the spectral coefficients of $f$ (they have to decrease to the order of machine precision, for double precision this is roughly $10^{-16}$ ) in domain I. We work in the following with the domains as before, I $(r<8)$, II $(8<$ $r<20)$ and III $(r>20)$ and use $N_{I}=40, N_{I I}=50$ and $N_{I I I}=20$ and $N_{x}=180$. The solution for $\omega=3 \mathrm{eV} / \hbar$ (a typical frequency considered in this context in nano-optics) can be seen in Fig. 10. The spectral coefficients of the solution in each domain in the lower row of the figure indicate that spectral accuracy is achieved.

For a dipole the strongest radiation is always expected orthogonal to the orientation of the dipole, here in the far field in the $\left\{x_{1}, x_{2}\right\}$ plane. The induced dipole moment in the nanoparticle can be read of at infinity. Since the leading contribution will be linear in $1 / r$ in this case, we simply differentiate $\mathcal{Y}$ in domain III with respect to $\rho=$ $1 / r$ and denote by $P$ the resulting value divided by $i k_{3}^{3} p$, the leading contribution of the dipole (51) at infinity. In Fig. 11 we plot this quantity for several values of $\omega$. The strongest resonance of the nanoparticle is observed for $\omega \approx 3 \mathrm{eV} / \hbar$.

\section{Conclusion}

In this paper we have presented a multi-domain spectral approach for the monochromatic Maxwell equations in an axisymmetric setting in spherical and prolate spheroidal coordinates. The Sommerfeld condition is imposed as in [5, 15, 16] exactly at infinity after splitting off an oscillatory factor. For several examples it is shown that machine precision can be reached with this approach. Obviously one could have 

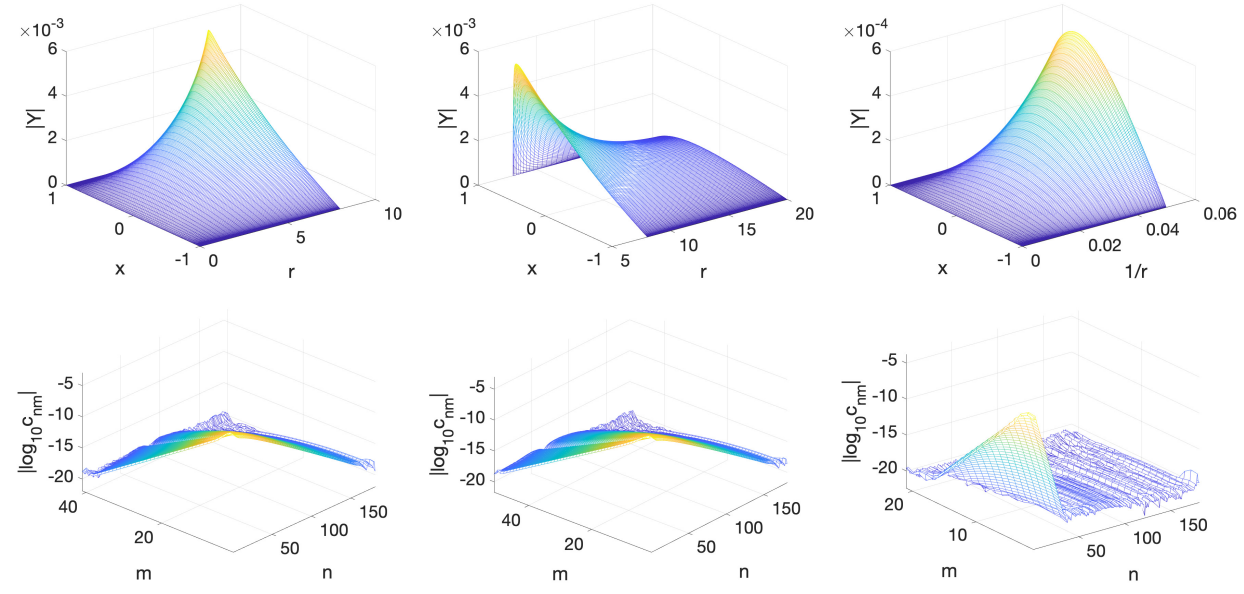

Figure 10. Solution to the equation (54) for $\omega=3 \mathrm{eV} / \hbar$ in the domains I, II, III (from left to right) in the upper row, and the corresponding spectral coefficients in the lower row.

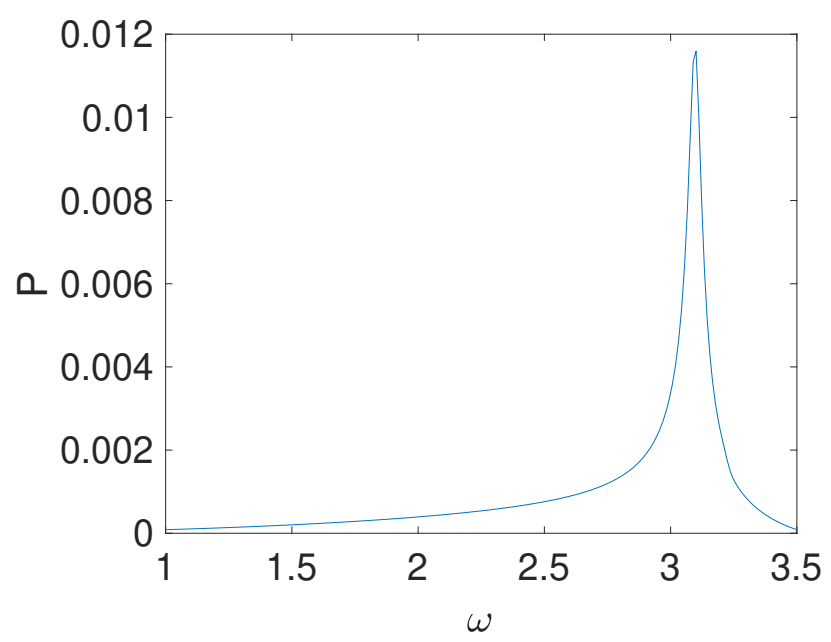

FIGURE 11. Induced dipole field in the nano-particle normalized by the dipole field at infinity in dependence of $\omega$.

built a similar spectral approach based on the eigenfunctions of the Helmholtz equation, in spherical coordinates Legendre polynomials and spherical Bessel functions. The differentiation matrices for Legendre polynomials are known, see for instance [30], but the spherical Bessel functions are transcendental functions that have to be computed as well. In particular the treatment of the Hankel function at infinity will need a similar treatment as presented here. In contrast to the case of Chebyshev polynomials, no fast algorithm to compute the spectral coefficients is known. The situation is worse in the prolate spheroidal case 
where the eigenfunctions are less well known. Thus it appears that the numerical method we discuss here could be also suitable to efficiently compute these functions which will be studied elsewhere along the lines of $[10]$.

Whereas we consider in this paper only the axisymmetric case, the approach is set up in a way that it can be extended to situations without symmetry. Instead of one twist potential, one has to deal with all three components of the electric field in this case in the Helmholtz equation (1). The discretization in $r$ and $x$ is as presented above. The dependence on the azimuthal coordinate $\phi$ can be addressed with a Fourier spectral method (see for instance [30]) which has the advantage of diagonal differentiation matrices. This means the equations decouple in $\phi$. For each of the $N_{\phi}$ collocation points in $\phi$, one thus has to solve a system for $\mathbf{E}$ with the methods discussed in the present paper. Since the equations do not couple in $\phi$, this is fully parallizable. A full $3 \mathrm{D}$ code along these lines will be the subject of further work.

\section{REFERENCES}

[1] Abramowitz, M., Stegun, I. (eds.): Handbook of Mathematical Functions with Formulas, Graphs, and Mathematical Tables. National Bureau of Standards (1970)

[2] F. V. Atkinson, On Sommerfeld's "Radiative Condition", Philos. Mag., 40 (1949), pp. 645-651.

BGT82 [3] A. Bayliss M. Gunzbergert, E. Turkel, Boudary conditions for elliptic equations in exterior regions, SIAM J. Appl. Math. Vol. 42, No.2 (1982)

birem [4] M. Birem and C. Klein, Multidomain spectral method for Schrödinger equations, Adv. Comp. Math., 42(2), 395-423 DOI 10.1007/s10444-015-9429-9 $(2016)$

BM81 [5] G. Beer, J. L. Meek, 'Infinite domain' elements, Num. Meth. in Engineering 17, 43-57 (1981)

BER94 [6] J.-P. Berenger, A perfectly matched layer for the absorption of electromagnetic waves, Journal of Computational Physics 114 (1994) 185 - 200.

[7] J.-P. Berrut, L.N. Trefethen, Barycentric Lagrange Interpolation, SIAM Rev. 46, No. 3, pp. 501-517 (2004).

[8] P. Bettess, Infinite Elements, Penshaw Press, Sunderland, 1992

[9] Clenshaw, C. W. and Curtis, A. R., A method for numerical integration on an automatic computer, Numer. Math. 2 (1960), 197-205.

[10] S. Crespo, M. Fasondini, C. Klein, N. Stoilov, C. Vallée, Multidomain spectral method for the Gauss hypergeometric function, Num. Alg., 84(1), 1-35 https://doi.org/10.1007/s11075-019-00741-7 (2019)

[11] D. Dzsotjan, B. Rousseaux, H. R. Jauslin, G. Colas des Francs, C. Couteau, and S. Guérin, Mode-selective quantization and multimodal effective models for spherically layered systems, Phys. Rev. A 94, 023818 (2016)

[12] T. Nozawa, N. Stergioulas, E. Gourgoulhon, Y. Eriguchi, Construction of Highly Accurate Models of Rotating Neutron Stars - Comparison of Three Different Numerical Schemes, Astron. Astrophys. 132(3) (1998) DOI: 10.1051/aas:1998304 
ECB14 [13] H. Espinoza R. Codinaa S. Badiaab, A Sommerfeld non-reflecting boundary condition for the wave equation in mixed form, Computer Methods in Applied Mechanics and Engineering Volume 276, 1 July 2014, Pages 122-148

joerg [14] J. Frauendiener, Conformal Infinity, Living Reviews in Relativity volume 3 (2000) https://doi.org/10.12942/lrr-2000-4

GJ17 [15] H. N. Gharti, J. Tromp, A spectral-infinite-element solution of Poisson's equation: an application to self gravity (2017) arxiv.org/1706.00855.jpg

GTZ18 [16] H. N. Gharti, J. Tromp, S. Zampini, Spectral-infinite-element simulations of gravity anomalies, Geophys. J. Int.215 (2018) 1098-1117

[17] H. N. Gharti, L. Langer, T. Jeroen, Spectral-infinite-element simulations of coseismic and post-earthquake deformation, Geophys. Jour. Int. 216(2) (2019), 1364-1393.

GLT2 [18] H. N. Gharti, L. Langer, T. Jeroen, Spectral-infinite-element simulations of earthquake-induced gravity perturbations, Geophys. Jour. Int. 217(1) (2019) 451-468.

[19] H. N. Gharti, T. Jeroen, Spectral-infinite-element simulations of magnetic anomalies, Geophys. Jour. Int. 217(1) (2019) 1656-1667.

GIV04 [20] D. Givoli, High-order local non-reflecting boundary conditions: a review, Wave Motion 39 (2004) 319 - 326.

GIV08 [21] D. Givoli, Computational absorbing boundaries, in: S. Marburg, B. Nolte (Eds.), Computational Acoustics of Noise Propagation in Fluids, volume 31, Springer Berlin Heidelberg, 2008, pp. 145-166.

KYK83 [22] Y. Kagawa, T. Yamabuchi, S. Kitagami, "The Infinite boundary element method and its application to a combined finite boundary element technique for unbounded field problems, COMPEL, Vol. 2 No. 4, pp. 179-193 (1983)

[23] C. Klein and O. Richter, Ernst Equation and Riemann Surfaces, Lecture Notes in Physics Vol. 685 (Springer) (2005).

[24] J.D. Jackson, Classical Electrodynamics John Wiley \& Sons Ltd. 1962

[25] C. Lanczos, Trigonometric interpolation of empirical and analytic functions, J. Math. and Physics, 17, 123-199 (1938)

[26] P. Moon, D.E. Spencer, Field Theory Handbook, Springer, Berlin 1988.

[27] J. Novak, S. Bonazzola, Absorbing boundary conditions for simulation of gravitational waves with spectral methods in spherical coordinates, J. Comp. Phys. 197(1) (2004), 186-196.

SHML [28] C. Sauvan, J. P. Hugonin, I.S. Maksymov, and P. Lalanne, Theory of the Spontaneous Optical Emission of Nanosize Photonic and Plasmon Resonators, Phys. Rev. Lett. 110, 237401 (2013)

SOM49 [29] A. Sommerfeld, Partial differential equations in physics, Elsevier, 1949.

[30] L. N. Trefethen, Spectral Methods in Matlab, SIAM, Philadelphia, PA, 2000.

[31] Trefethen, L.N., 2013. Approximation theory and approximation practice (Vol. 128). Siam.

T98 [32] S. V. Tsynkov, Numerical solution of problems on unbounded domains. A review, Applied Numerical Mathematics 27 (1998) 465 - 532.

[33] Weideman, J.A.C. and Reddy, S.C., A Matlab differentiation matrix suite, ACM TOMS, 26 (2000), 465-519.

WIL [34] C.H. Wilcox, A generalization of theorems of Rellich and Atkinson, Proc. Amer. Math. Soc., (1955), pp. 271-276.

lorene [35] www.lorene.obspm.fr

VP12 [36] C. van Vlack and P. Hughes, Spontaneous emission spectra and quantum lightmatter interactions from a strongly coupled quantum dot metal-nanoparticle system, Phys. Rev. B 85, 075303 (2012) 
ZKB79 [37] O.C. Zienkiewicz, I.D.W. Kelly, P. Bettess, The Sommerfeld radiation condition on the infinite domains and its modeling in numerical procedures, Computing methods in applied sciences and engineering, Amsterdam 1979

Institut de Mathématiques de Bourgogne, UMR 5584, Université De Bourgogne-Franche-Comté, 9 avenue Alain Savary, 21078 Dijon Cedex, France, E-mail Christian.Klein@u-Bourgogne.Fr

Institut de Mathématiques de Bourgogne, UMR 5584, Université De Bourgogne-Franche-Comté, 9 avenue Alain Savary, 21078 Dijon Cedex, France, E-Mail Nikola.Stoilov@u-Bourgogne.Fr 